\section{Human platelets are a source of collagen I}

Platelets are involved in primary hemostasis but also affect or modulate inflammatory and immune responses as well as angiogenesis ${ }^{1}$ and cancer metastasis. ${ }^{2}$ Activated platelets can affect the function of other cells either through direct cell-cell interaction or through the release of soluble factors and microparticles. Moreover, despite being anucleate, platelets contain mRNA and are able to synthesize proteins. ${ }^{3}$ However, the platelet protein land- scape has not been fully explored and there is evidence of dynamic changes in the platelet proteome upon activation. To investigate potential mechanisms underlying platelet hyper-reactivity, platelets from healthy subjects and patients with polycystic ovary syndrome (PCOS) were studied. The latter syndrome is a common endocrinopathy affecting women of reproductive age and is associated with abnormal platelet responses. ${ }^{4}$ Here we report, for the first time, that human platelets express pro-collagen I mRNA and protein, which is secreted in response to platelet activation and processed by throm-
A

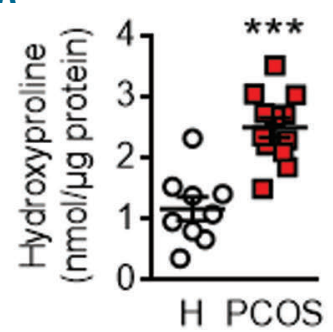

D

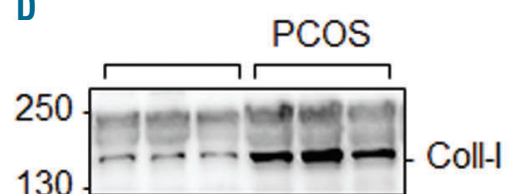

36 .
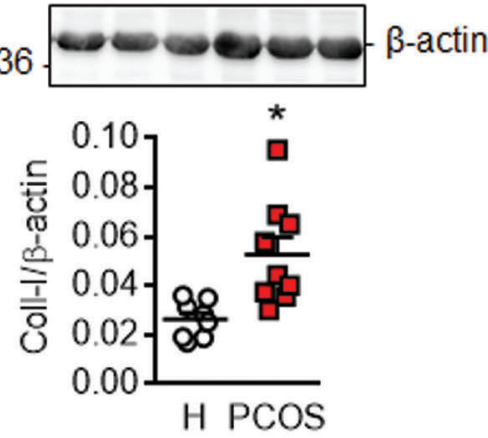

B

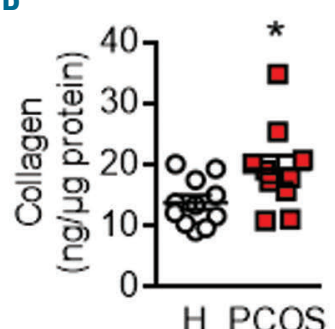

C

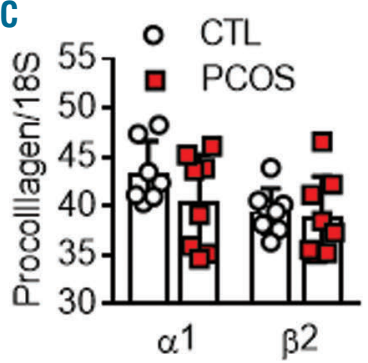

E
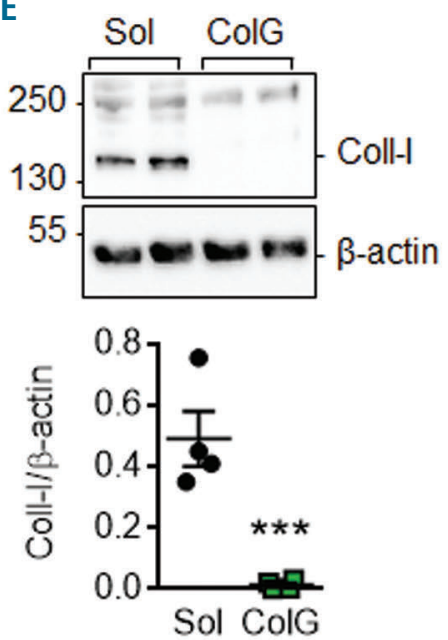

$\mathrm{F}$

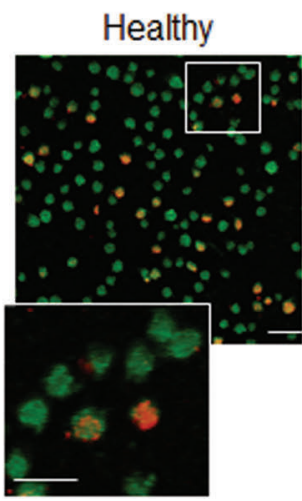

PCOS

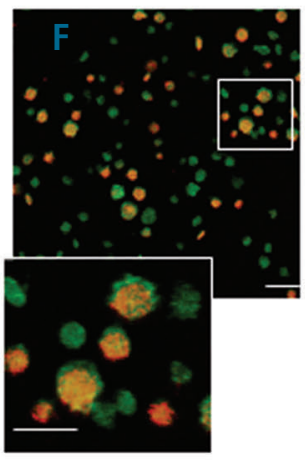

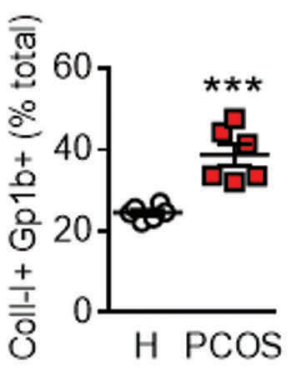

Collagen I Gp1b

Figure 1. Hydroxyproline and collagen levels in human platelets. (A) Hydroxyproline levels in platelets from healthy donors $(\mathrm{H})$ and polycystic ovary syndrome (PCOS) subjects, $\mathrm{n}=9-13$ (Student's t-test, $* * * P<0.001$ ). (B) Collagen levels in platelets from healthy donors $(H)$ and from PCOS subjects, $\mathrm{n}=10$ (Student's $t$-test, $* P<0.05$ ). (C) Expression of mRNA encoding collagen I $\alpha 1$ and $\beta 2$ in platelets from healthy donors and PCOS patients, $n=7-8$ (ANOVA, Tukey's multiple comparisons post test, $P>0.1$ ). (D) Representative blots (upper panel) and quantification (lower panel) showing collagen I $\alpha 1$ subunit in platelets from $\mathrm{H}$ and PCOS subjects, $\mathrm{n}=5-7$ (Student's t-test, $* P<0.05$ ). (E) Representative blots (upper panel) and quantification (lower panel) showing collagen I $\alpha 1$ expression in platelet lysates from PCOS subjects treated with solvent or collagenase (ColG), $\mathrm{n}=4$ (Student's $t$-test, $* * * P<0.001)$. (F) Representative images (left panel) and quantification (right panel) of collagen I (red) and Gp1b (green) in unstimulated non-permeabilized platelets from $\mathrm{H}$ or PCOS subjects; bar $=10 \mu \mathrm{m}$; $\mathrm{n}=6$ (Student's $t$-test, $* * * P<0.001)$. 
A

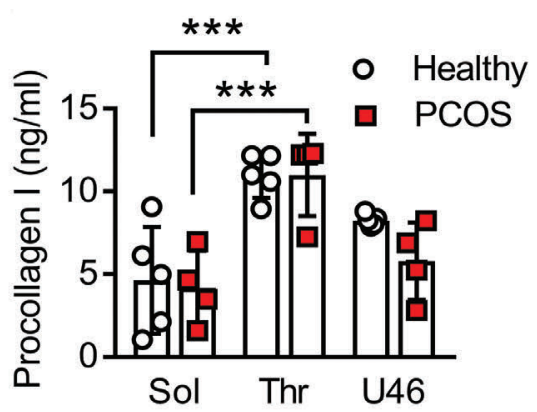

C

Sol ᄀ־ UK ᄀ

Sol 1060 Sol $1060 \mathrm{Thr}(\mathrm{min})$

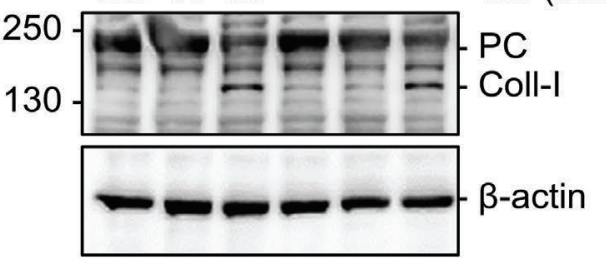

D
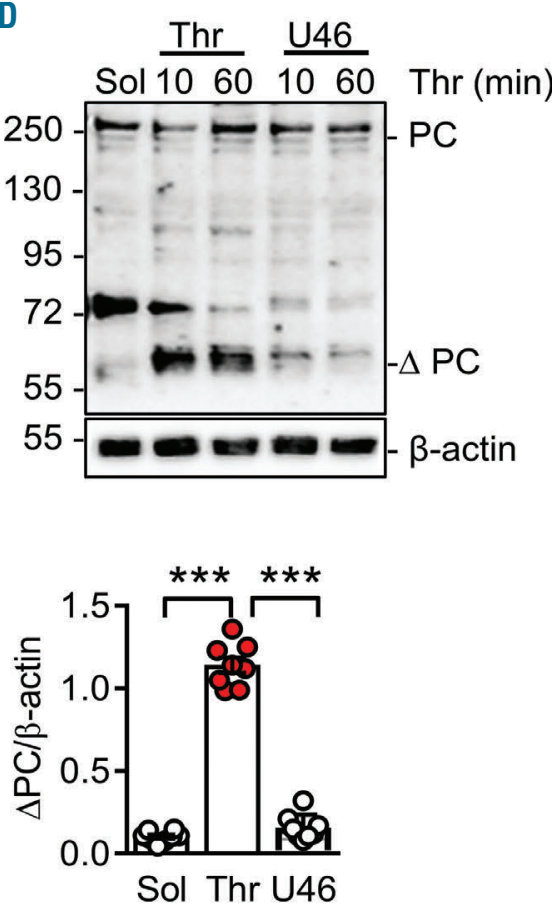

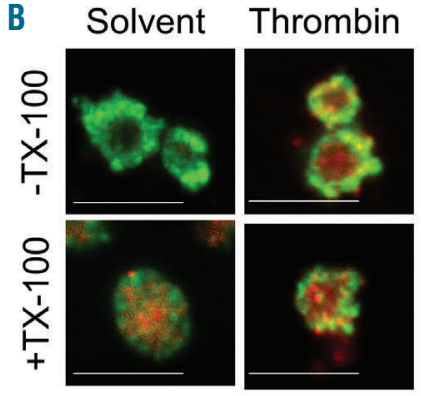

Gp1b Pro-collagen

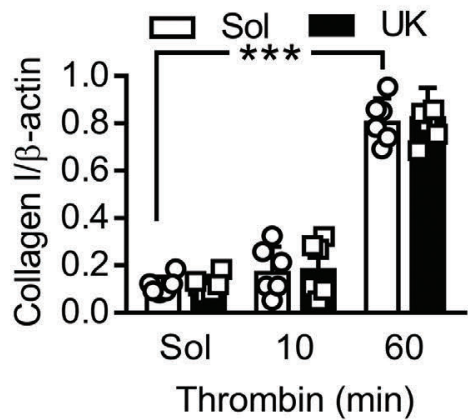

E
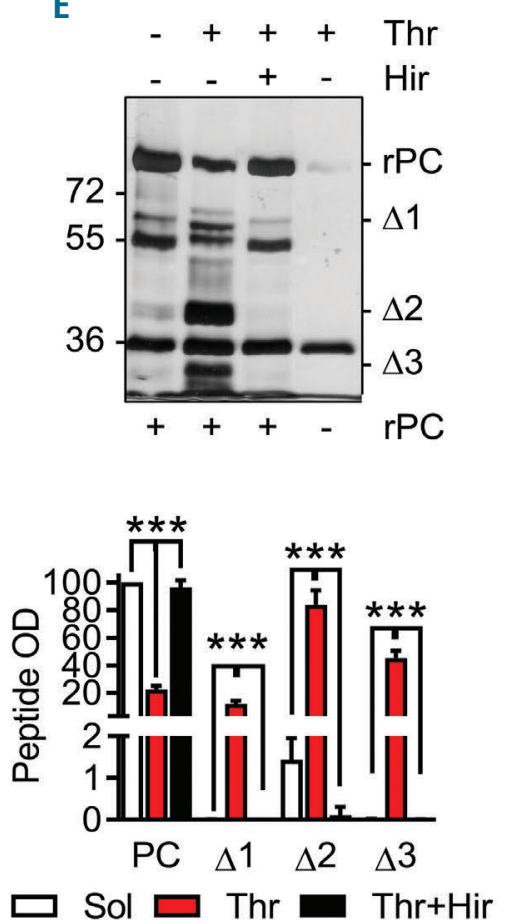

Figure 2 (previous page). Release of pro-collagen by activated platelets and processing by thrombin. (A) Procollagen released from platelets from healthy donors or polycystic ovary syndrome (PCOS) patients treated with solvent (Sol), thrombin (Thr) $(1 \mathrm{U} / \mathrm{mL}, 10 \mathrm{minutes})$ or the thromboxane A2 analog U46619 (U46, 1 umol/L, 10 minutes); $n=4-5$ (ANOVA and Tukey's multiple comparisons post test, ***P<0.001). (B) Pro-collagen I (red), and Gp1b (green) expression in non-permeabilized (-TX-100) and permeabilized (+TX-100) platelets from healthy donors, treated with Sol or Thr (1U/mL, 10 minutes); bar $=5 \mu \mathrm{m}$. Comparable results were obtained in five additional experiments. (C) Representative blots (left panel) and quantification (right panel) showing collagen I in platelets from healthy donors stimulated with either Sol or Thr in the presence or in the absence of the BMP inhibitor UK383367 (UK), $\mathrm{n}=6$ (ANOVA and Tukey's multiple comparisons post test, $* * * P<0.001$ ). (D) Representative blot (upper panel) and quantification (lower panel) showing pro-collagen (PC) and its cleavage product $(\triangle P C)$ in platelets treated with Sol, Thr (1U/mL) or U46619 (U46, 1 $\mu \mathrm{mol} / \mathrm{L}$ ) for up to 60 minutes. The bar graph shows the quantification of cleaved pro-collagen $(\triangle \mathrm{PC})$ after 60 minutes, $\mathrm{n}=8$ (ANOVA and Tukey's multiple comparisons post test, $* * * P<0.001$ ). (E) Representative silver gel (upper panel) and quantification (lower panel) showing the cleavage of a 80 $\mathrm{kD}$ recombinant pro-collagen $(\mathrm{rPC})$ by Thr) $(1 \mathrm{U} / \mathrm{mL}, 30$ minutes $)$ in the absence or in the presence of hirudin $(\mathrm{Hir})(1 \mathrm{U} / \mathrm{mL}), \mathrm{n}=4 \mathrm{independent}$ experiments (ANOVA and Tukey's multiple comparisons post-test, $* * * P<0.001$ ). 
bin to mature collagen I, which then binds to platelet membrane and decorates the platelet surface. The experimental evidence relating to the expression and processing of collagen I is briefly presented here; the wider implications these findings can have for PCOS patients and other metabolic conditions are the subject of a separate study.

Amino acid profiling revealed that platelets from PCOS patients contained significantly more hydroxyproline than platelets from non-PCOS subjects (Figure 1A), with no consistent differences in levels of the other amino acids evaluated (see Online Supplementary Table S1 for amino acid concentrations and Online Supplementary Figure S1 for the heatmap). Hydroxyproline is a proteinogenic amino acid that is produced by the hydroxylation of proline and a major component of fibrillary collagen, 5,6 making it a reliable indicator of collagen levels. As platelets are known to respond to collagen, ${ }^{7}$ but not to generate it, different techniques were used to verify platelet expression of collagen. Using an assay based on the acid hydrolysis of samples to form hydrolysates and hydroxyproline, that is specific for collagen, it was possible to confirm that platelets express collagen. Moreover, platelets from the PCOS collective expressed significantly more collagen than platelets from healthy individuals (Figure 1B). Mass spectrometry identified collagen I, particularly the $\alpha 1$ and $\alpha 2$ subunits, as the most abundant isoform (Table 1). This fits well with the detection of mRNA encoding pro-collagen I $\alpha 1$ and $\alpha 2$ in human platelets (Figure 1C), implying that collagen I could be synthesized de novo by platelets. Western blotting confirmed the expression of collagen in platelets and revealed that the platelet activation associated with PCOS increased platelet collagen I expression (Figure $1 D)$, even though mRNA levels were comparable in platelets from the healthy and PCOS donors. The anticollagen I antibody used did not bind to samples that had been pre-treated with collagenase, confirming the specific binding of the antibody to collagen I (Figure 1E). Finally, immunohistochemistry of platelets fixed on polyL-lysine confirmed the presence of collagen I on the surface of non-permeabilized platelets (Figure 1F).

Collagens are synthesized from pro-collagens that are secreted into the extracellular space where they undergo proteolysis to remove the $\mathrm{N}$ - and $\mathrm{C}$-terminal extension peptides before being cross-linked and assembled into collagen fibrils. ${ }^{8}$ We next assessed whether platelets are able to release pro-collagen I and to process it into mature collagen I. Procollagen I was detectable in the supernatant of unstimulated platelets but levels increased significantly after stimulation with thrombin (Figure 2A) whereas platelet stimulation with the thromboxane A2 analog, U46619, exerted a much weaker effect. No significant differences were observed between platelets from healthy and from PCOS subjects, suggesting the storage of similar amounts of pro-collagen in platelets from healthy and PCOS patients. These findings implied that the higher levels of mature collagen I detected in platelets from PCOS patients may be linked to the accelerated conversion of pro-collagen I to mature collagen I. Indeed, in the absence of a platelet agonist, pro-collagen I was only detected in Triton X-100 permeabilized platelets, i.e., pro-collagen I was stored and sequestered intracellularly. However, after stimulation with thrombin, pro-collagen was released to decorate the platelet surface and could be detected in the absence of Triton X-100 (Figure $2 \mathrm{~B})$. The finding that platelets express pro-collagen I mRNA and are able to release pro-collagen I protein in response to platelet agonists convincingly demonstrates
Table 1. Collagen isoforms detected in human platelets.

\begin{tabular}{lccc}
\hline Protein name & Gene name & Peptides & Uniprot ID \\
Collagen alpha-2(I) chain & COL1A2 & 5 & P08123 \\
Collagen alpha-1(I) chain & COL1A1 & 2 & P02452 \\
\hline Collagen alpha-1(XII) chain & COL12A1 & 1 & H0Y5N9 \\
Collagen alpha-1(III) chain & COL3A1 & 1 & P02461 \\
\hline
\end{tabular}

that platelets are a novel source of pro-collagen I. Although the presence of enzymes involved in collagen biosynthesis in human platelets was reported more than 40 years ago, ${ }^{9}$ this study presents the first evidence of pro-collagen I release by platelets and the presence of mature collagen on the platelet surface.

To determine whether pro-collagen I could be converted to collagen I upon platelet stimulation, platelets from healthy donors were stimulated with thrombin and levels of collagen were assessed by western blotting. This revealed a clear thrombin-induced decrease in pro-collagen I and increase in collagen I levels suggesting that collagen I was generated in response to platelet activation (Figure 2C). A number of different peptidases have been implicated in the generation of collagen, ${ }^{10}$ including bone morphogenic protein (BMP)-1 which is the main pro-collagen C-proteinase. ${ }^{11}$ There is no evidence of BMP1 expression in platelets (PlateletWeb database ${ }^{12}$ ) and the BMP-1 inhibitor; UK383367, failed to affect the thrombin-induced increase in collagen I levels. These observations suggested that an alternative protease could target platelet pro-collagen I, and as thrombin was suggested to cleave pro-collagen in cultured fibroblasts, ${ }^{13}$ collagen cleavage was studied in platelets stimulated with thrombin or the thromboxane A2 analog. Thrombin and U46619 both elicit platelet activation; however, only thrombin led to the cleavage of pro-collagen and the appearance of a $\sim 60 \mathrm{kD}$ product recognized by the procollagen antibody (Figure 2D). To confirm this finding, a truncated recombinant pro-collagen $\alpha 1(\sim 80 \mathrm{kD})$ that is a substrate for procollagen $\mathrm{N}$ - and C-proteinases was incubated with thrombin in vitro. This procedure resulted in the appearance of three additional peptides $(\sim 60, \sim 40$ and $\sim 28 \mathrm{kD}$ ), and was prevented by the thrombin inhibitor, hirudin (Figure 2E).

Taken together, the results of the present study demonstrate that platelets are a source of circulating collagen. Using different techniques, i.e., mass spectrometry, a specific assay for total collagen, real-time-quantitative polymerase chain reaction, immunoblotting and immunohistochemistry, it was possible to show that platelets express pro-collagen I mRNA and protein. The latter is secreted in response to platelet activation and can undergo thrombin-mediated proteolysis to generate mature collagen I that binds to the platelet surface. The platelet receptor that binds pro-collagen I was not characterized here, but procollagen I very likely attaches to platelets via $\alpha 2 \beta 1$ integrin. ${ }^{14}$ The functional consequences of the enhanced collagen I levels on the surface of the pre-activated platelets from PCOS patients remains to be demonstrated. However, the collagen-coating on activated platelets may well act as an agonist for other cells, to link platelet activation with the enhanced atherothrombosis risk in these patients. ${ }^{15}$

Anastasia Kyselova, 1,2 Sven Zukunft, 1,2 Deborah Puppe, Ilka Wittig, ${ }^{2,3}$ W. Alexander Mann, ${ }^{4}$ Imke Dornauf, ${ }^{4}$ Ingrid Fleming ${ }^{1,2}$ and Voahanginirina Randriamboavonjy $y^{1,2}$

${ }^{1}$ Institute for Vascular Signalling, Centre of Molecular Medicine, 
Goethe University, Frankfurt am Main; ${ }^{2}$ German Center of Cardiovascular Research (DZHK), Partner site Rhein Main, Frankfurt am Main; ${ }^{3}$ Functional Proteomics, SFB 815 Core Unit, Faculty of Medicine, Goethe Universitv, Frankfurt am Main and ${ }^{4}$ Endokrinologikum Frankfurt, Frankfurt am Main, Germany.

Correspondence:

VOAHANGINIRINA RANDRIAMBOAVONJY

voahangy@vrc.uni-frankfurt.de

doi:10.3324/haematol.2020.255612

Disclosures: no conflicts of interest to disclose.

Contributions: $A K, D P, I W$ and $S Z$ performed experiments, acquired the data and analyzed results; WAM and ID characterized the patients; VR conceived and designed the research and wrote the manuscript; IF co-designed the study and wrote the manuscript.

Funding: this work was supported by the Deutsche Forschungsgemeinschaft (RA 2435/3-2 to VR and FL 364/7-1 to IF).

\section{References}

1. Smyth SS, McEver RP, Weyrich AS, et al. Platelet functions beyond hemostasis. J Thromb Haemost. 2009;7(11):1759-1766.

2. Schumacher D, Strilic B, Sivaraj KK, Wettschureck N, Offermanns S. Platelet-derived nucleotides promote tumor-cell transendothelial migration and metastasis via P2Y2 receptor. Cancer Cell. 2013; 24(1):130-137.

3. Beaulieu LM, Freedman JE. NFkappaB regulation of platelet function: no nucleus, no genes, no problem? J Thromb Haemost. 2009, 7(8):1329-1332.

4. Randriamboavonjy V, Mann WA, Elgheznawy A, et al. Metformin reduces hyper-reactivity of platelets from patients with polycystic ovary syndrome by improving mitochondrial integrity. Thromb Haemost. 2015;114(3):569-578.

5. Ogle JD, Arlinghaus RB, Lgan MA. 3-Hydroxyproline, a new amino acid of collagen. J Biol Chem. 1962;237:3667-3673.

6. Udenfriend S. Formation of hydroxyproline in collagen. Science. 1966;152(3727):1335-1340

7. Nuyttens BP, Thijs T, Deckmyn H, Broos K. Platelet adhesion to collagen. Thromb Res. 2011;127(Suppl 2):S26-S29.

8. Last JA, Reiser KM. Collagen biosynthesis. Environ Health Perspect. 1984:55:169-177.

9. Anttinen H, Tuderman L, Oikarinen A, Kivirikko KI. Intracellular enzymes of collagen biosynthesis in human platelets. Blood. 1977;50(1):29-37

10. Prockop DJ, Sieron AL, Li SW. Procollagen N-proteinase and procollagen C-proteinase. Two unusual metalloproteinases that are essential for procollagen processing probably have important roles in development and cell signaling. Matrix Biol. 1998;16(7):399-408.

11. Garrigue-Antar L, Barker C, Kadler KE. Identification of amino acid residues in bone morphogenetic protein-1 important for procollagen C-proteinase activity. J Biol Chem. 2001;276(28):26237-26242.

12. Boyanova D, Nilla S, Birschmann I, Dandekar T, Dittrich M PlateletWeb: a systems biologic analysis of signaling networks in human platelets. Blood. 2012;119(3):e22-e34.

13. Keski-Oja J, Todaro GJ, Vaheri A. Thrombin affects fibronectin and procollagen in the pericellular matrix of cultured human fibroblasts. Biochim Biophys Acta. 1981;673(3):323-331.

14. Weston SA, Hulmes DJ, Mould AP, Watson RB, Humphries MJ. Identification of integrin alpha 2 beta 1 as cell surface receptor for the carboxyl-terminal propeptide of type I procollagen. J Biol Chem. 1994:269(33):20982-20986.

15. Randeva HS, Tan BK, Weickert MO, et al. Cardiometabolic aspects of the polycystic ovary syndrome. Endocr Rev. 2012;33(5):812-841. 